\title{
SÍNDROME PARSONAGE-TURNER: a propósito de un caso de omalgia atraumática.
}

\author{
Sanchez-Ramos Caballero E ${ }^{1}$, Martín Escartín $C^{2}$, Real Collado C ${ }^{1}$, Córdoba Félix J ${ }^{1}$, Navarro Calvo A ${ }^{2}$. \\ 1 Servicio de Medicina Física y Rehabilitación, Hospital Clínico, Valencia \\ 2 Servicio de Medicina Física y Rehabilitación, Hospital Arnau Vilanova, Valencia. \\ * Correspondencia: eduardosrc93@gmail.com
}

Academic Editor: Firstname

Lastname

Received: date

Accepted: date

Published: date

Copyright: (c) 2021 by the authors. Submitted for possible open access publication under the terms and conditions of the Creative Commons Attribution (CC BY) license (http://creativecommons.org/licenses /by/4.0/).

\begin{abstract}
El Síndrome de Parsonage-Turner (SPT), también conocido como neuralgia amiotrófica, se caracteriza por un dolor de inicio agudo localizado en la zona proximal del miembro superior, que evoluciona presentando paresias musculares, atrofias y alteraciones de la sensibilidad. Para el diagnóstico de la patología es fundamental realizar una anamnesis y exploración minuciosa, y pueden ser de gran utilidad pruebas complementarias como la resonancia magnética y electromiografía, que nos permitan llegar a esta enfermedad como diagnóstico de exclusión. Es importante realizar un abordaje multidisciplinar de esta entidad tanto desde el punto de vista diagnóstico como terapéutico. Tanto el diagnóstico como el tratamiento farmacológico y rehabilitador deben ser precoces para mejorar el pronóstico.
\end{abstract}

Keywords: Parsonage-Turner syndrome, atraumatic shoulder pain, muscle paresis.

\section{Introducción}

La neuralgia amiotrófica, también conocida como síndrome de Parsonage-Turner o neuritis braquial es una enfermedad con una incidencia aproximada de 1,6 casos/100.000 habitantes ${ }^{1}$, con predominio en el sexo masculino ${ }^{2}$, que se manifiesta como una neuropatía aguda del plexo braquial $^{3}$. Se trata de un proceso inflamatorio multifocal, inmunomediado, que involucra los nervios periféricos, y cuya etiología y fisiopatología se desconocen actualmente ${ }^{4,5}$. Se han descrito factores precipitantes como infecciones, traumatismos, ejercicio físico intenso, cirugías, partos traumáticos o enfermedades sistémicas ${ }^{6}$.

Clínicamente se caracteriza por presentar varias fases. La fase inicial, que dura aproximadamente 3 semanas, se caracteriza por la parición de un dolor de inicio brusco en las extremidades superiores, pulsátil y de predominio nocturno, seguido por una fase atrófica con presencia de debilidad, pérdida de masa muscular, acompañada de déficits neurológicos progresivos y, en ocasiones, déficits sensitivos 7,8. Hasta el $80 \%$ de los pacientes tienen un inicio unilateral, y en el $60 \%$ de los casos se afecta el lado dominante 9 . 
Durante la fase atrófica existe compensación por parte de se producen estrategias compensatorias por parte de los músculos estabilizadores del hombro, que suelen derivar en una discinesia escapular ${ }^{10}$.

La afectación del plexo implica más comúnmente a los troncos superiores y medios, siendo los nervios motores los que se afectan con mayor frecuencia, entre los que destacan el nervio torácico largo, supraescapular, axilar, musculocutáneo, interóseo anterior y posterior ${ }^{5}$.

En cuanto al diagnóstico, se basa en la realización de una anamnesis y exploración físicaminuciosas que evidencien los síntomas anteriormente mencionados. Además, es aconsejable realizar una resonancia magnética del hombro afectado y la columna cervical, que a pesar de mostrar una imagen inespecífica ${ }^{11,12}$, nos permite descartar otras enfermedades como la rotura del manguito de los rotadores, plexopatía traumática, daño del labrum, síndrome de atrapamiento y tendinitis cálcica 7,9 . También se considera útil la realización de electromiografía, la cual evidenciará una afectación motora considerable ${ }^{13}$.

No existe un tratamiento específico para la neuralgia amiotrófica, pero es posible conseguir una recuperación completa en más de la mitad de los pacientes en un periodo de dos a tres años, dependiendo de la gravedad de los síntomas iniciales ${ }^{14}$.

La mayoría de los pacientes con SPT son tratados con un enfoque multidisciplinar que incluye tanto tratamiento farmacológico como terapias físicas ${ }^{15}$. Sin embargo, existen casos en los que no es posible recuperar la función muscular evidenciándose la persistencia de un déficit neurológico.

\section{Caso clínico}

Paciente varón de 81 años, con antecedentes de Enfermedad Pulmonar Obstructiva Crónica (EPOC), dislipemia e hipertensión arterial que acude al Servicio de urgencias por omalgia izquierda de 3 semanas de evolución de inicio súbito, sin antecedente traumático. Presenta dolor de características neuropáticas con irradiación hasta el primer dedo mano izquierda y con un valor de 8/10 en la Escala Visual Analógica (EVA), que empeora con las movilizaciones pasivas del hombro.

A la exploración física destaca una cervicalgia irradiada a hombro izquierdo con un balance muscular (BM) de miembro superior izquierdo (MSI) de 5/5 excepto el bíceps braquial donde la fuerza es de 1/5 y una hipoestesia marcada en territorio C5-C6-C7. debilidad del MSI y de la cintura escapular, principalmente con debilidad para la flexión del codo.

Ante la sospecha de un SPT, se deriva a consultas externas, donde se realiza el diagnóstico diferencial con enfermedades neoplásicas, infecciosas o autoinmunes y se solicita una resonancia magnética (RM) cervical y de hombro y una electromiografía (EMG).

En la RM se objetiva una estenosis del canal cervical sin mielopatía, pinzamiento del espacio graso subacromial y rotura completa del tendón supraespinoso (Figura 1). 


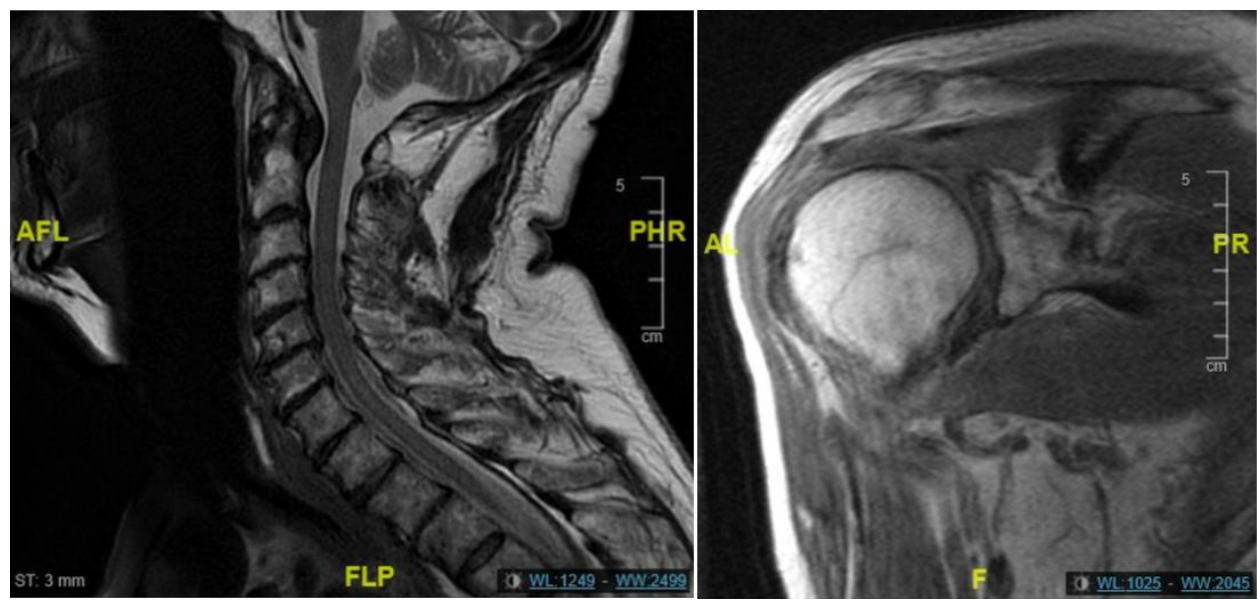

Figura 1: Imágenes de RM cervical y RM de hombro izquierdo.

Por otro lado, en la EMG se evidencia una plexopatía braquial izquierda con mayor afectación en la división anterior del tronco superior (nervio musculocutáneo) de grado grave y de una afectación de las porciones anterior y posterior del tronco medio (nervio mediano y nervio radial) de grado moderado (Figura 2) que muestra ausencia de potenciales de respuesta sensitiva (PRS) en los dermatomas C6 y C7 (Figura 3). En la división anterior del tronco superior (nervio mediano) presenta una pérdida masiva de unidades motoras no activa y con signos de reinervación subaguda (Figura 4).

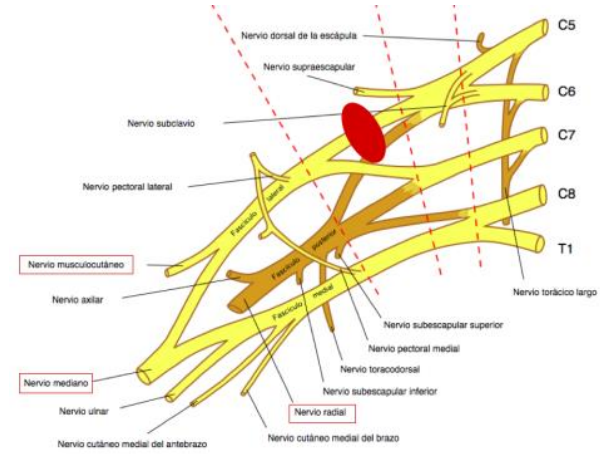

Figura 2: Anatomía del plexo braquial y localización de la lesión.

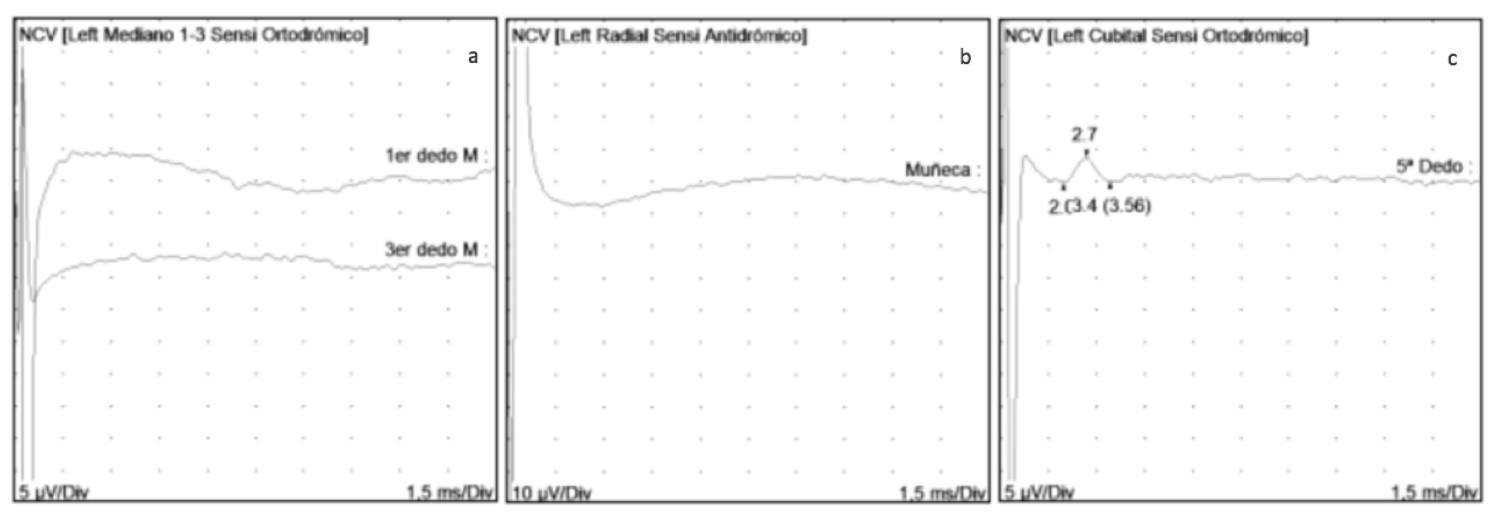

Figura 3: Imagen ausencia de conducciones sensitivas de los nervios mediano y radial (a, b), conducciones del nervio cubital normal (c). 


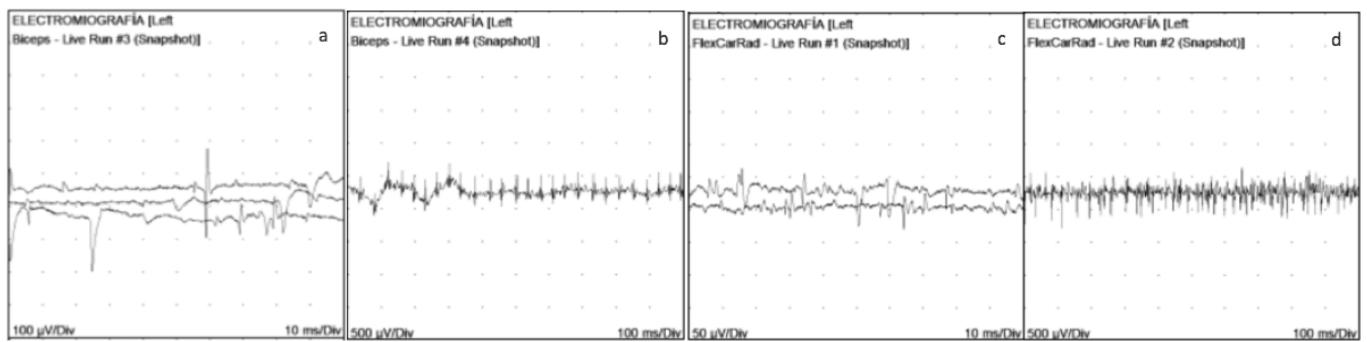

Figura 4: Afectación de la división anterior del tronco superior (biceps y carpis radialis) que muestran una denervación activa con ondas bifásicas y ondas positivas $(a, c)$ y un trazado simple (b, d).

Tras realizar el diagnóstico de exclusión, se decide iniciar tratamiento con corticoides a dosis altas, analgesia y tratamiento rehabilitador que consiste en ganancia de recorrido articular mediante movilizaciones activo-asistidas del miembro superior, potenciación musculatura flexora del codo, electroterapia (corrientes interferenciales) y ejercicios de propiocepción.

Durante el tratamiento, el paciente ganó de forma progresiva fuerza en el MSI hasta alcanzar un BM de 5/5, mejoró la sincinesia escapular y recuperó parcialmente la sensibilidad, persistiendo una hipoestesia leve en el territorio de C7.

Una vez finalizado el tratamiento se solicitó un EMG de control donde se evidenció una plexopatía braquial izquierda de la división anterior de los troncos superior y medio, de predominio sensitivo, con una afectación actual leve de las fibras motoras, observando un aumento de la densidad en el trazado al máximo esfuerzo y la presencia de unidades motoras polifásicas e inestables que demuestran un progreso en la reinervación.

\section{Discusión}

La omalgia sin traumatismo previo es uno de los principales motivos de consulta de Traumatología, Rehabilitación, Neurología y Reumatología. Por ello, es conveniente la realización de una anamnesis y exploración física minuciosas a fin de establecer un diagnóstico diferencial adecuado ${ }^{9}$.

Ante un paciente con omalgia asociada a alteraciones de la sensibilidad y/o paresias musculares es importante tener en cuenta el Síndrome de Parsonage-Turner, ya que se trata de una patología infradiagnosticada que cursa con una clínica poco específica y por consiguiente asocia un retraso en el diagnóstico ${ }^{6,9}$.

Es necesario solicitar pruebas complementarias como la EMG y la RM para ayudar en el diagnóstico en un contexto clínico adecuado. Si bien el SPT se considera un diagnóstico de exclusión, los hallazgos en la EMG mostrarán una denervación aguda proveniente de una neuropatía axonal ${ }^{13}$.

La RM a pesar de no ser específica, nos permite observar en fases iniciales el edema muscular y posteriormente la atrofia del músculo correspondiente a la denervación, dándonos información sobre el estado evolutivo y ayudando su diagnóstico diferencial ${ }^{11}$, 12.

El diagnóstico precoz del SPT es determinante en la recuperación completa del paciente, por lo que debemos incluirlo en el diagnóstico diferencial del hombro doloroso sin antecedente traumático para evitar el retraso del inicio del tratamiento. 
No disponemos de un tratamiento específico para esta neuropatía, que en ocasiones es resistente al tratamiento farmacológico, por ello es importante iniciar de forma precoz el tratamiento rehabilitador con el objetivo de mantener el recorrido articular del hombro y recuperar la fuerza muscular ${ }^{15}$.

El tiempo hasta la recuperación completa es variable, pudiendo presentar síntomas residuales como dolor, fatiga muscular en hombro y cuello y discinesia escapular a pesar de la recuperación del nervio periférico, ya que siguen sin evidenciarse los mecanismos cerebrales que sugieren dicha afectación ${ }^{14,15}$.

\section{Conclusiones}

Es necesario enfatizar sobre la necesidad de tener presente esta patología en el diagnóstico diferencial de la omalgia sin historia previa de traumatismo. Teniendo en cuenta, que el Síndrome Parsonnage Turner o Neuralgia amiotrófica es una entidad clínica dolorosa de difícil diagnóstico mediante pruebas complementarias, por lo que se ha de considerar el diagnóstico diferencial de otras patologías del hombro para poder establecer un diagnóstico de exclusión de ésta.

A su vez, a mayor brevedad en el inicio de tratamiento conseguiremos un mejor pronóstico, así como una reducción considerable en el número de complicaciones.

\section{Referencias bibliográficas}

1. Cepillo AJ, Martín-Tamayo P, Mayordomo P, Onsurbe M, Toledo I, García DO. Una rareza infradiagnosticada: síndrome de Parsonage-Turner (neuritis braquial aguda). Rev Pediatr Aten Primaria. 2012;14:145-8.

2. Valle N, Aldaz I, Sánchez G, Martín I, Herrero A, Síndrome de Parsonage Tuerner; a propósito de dos casos en atención primaria. Semergen. 2010;36(7):406-408. DOI: 10.1016/j.semerg.2010.03.003

3. L. Muñoz-Cabello, C. López-Figueres, M.T. Rueda-Zafra, F.J. Mayordomo-Riera, Síndrome de Parsonage-Turner en paciente trasplantado de pulmón. A propósito de un caso.Rehabilitación,Volume 51, Issue 4,2017,Pages 273-276,ISSN 00487120, https://doi.org/10.1016/j.rh.2017.09.007.

4. Katzer A, Niedermauntel WP, Rump J. Das Parsonage-Turner-Syndrom [Parsonage-Turner Syndrome - Case Report]. Z Orthop Unfall. 2017;155(6):705-707

5. Al Khalili Y, Jain S, DeCastro A. Brachial Neuritis. In: StatPearls. Treasure Island (FL): StatPearls Publishing; 2020.

6. C.M. Martínez Bermúdez, I. Fernández de Gea, B. Yusá Cubes, H. Valera Martínez. Parsonage-Turner syndrome: Unknown but not infrequent. Apropos of 6 cases. Servicio de Medicina Física y Rehabilitación, Hospital Clínico Universitario Virgen de la Arrixaca, Murcia,Vol. 53. Núm. 1.páginas 56-59 . DOI: 10.1016/j.rh.2018.05.001

7. Smith CC, Bevelaqua AC. Challenging pain syndromes: Parsonage-Turner syndrome. Phys Med Rehabil Clin N Am. 2014;25(2):265-277

8. Joaquim AF, Makhni MC, Riew KD. Post-operative nerve injuries after cervical spine surgery. Int Orthop. 2019;43(4):791795.

9. Francisco Briceño Procopioa, Sergio A. Rodríguez Montero. Síndrome de Parsonage-Turner. Revisión bibliográfica. Semin Fund Esp Reumatol. 2010:11(4): 144-151, DOI: 10.1016/j.semreu.2010.05.002

10. Lustenhouwer R, van Alfen N, Cameron IGM, et al. NA-CONTROL: a study protocol for a randomised controlled trial to compare specific outpatient rehabilitation that targets cerebral mechanisms through relearning motor control and uses selfmanagement strategies to improve functional capability of the upper extremity, to usual care in patients with neuralgic amyotrophy. Trials. 2019;20(1):482.

11. Scalf RE, Wenger DE, Frick MA, Mandrekar JN, Adkins MC. MRI findings of 26 patients with Parsonage-Turner syndrome. AJR Am J Roentgenol. 2007;189:W39-44.

12. Shaukat MFS, Ogunwale B, Arpan BK. MRI Appearances of Parsonage-Turner Syndrome. J Coll Physicians Surg Pak. 2019;29(11):1127-1128. 
13. Seror P. Neuralgic amyotrophy. An update. Joint Bone Spine. 2017;84:153-8. Disponible en: https://doi.org/10.1016/j.jbspin.2016.03.005.

14. Favero KJ, Hawkins RH, Jones MW, Neuralgic amyotrophy. J Bone Joint Surge Br. 1987; 69: 195-8.

15. Janssen RMJ, Satink T, Ijspeert J, et al. Reflections of patients and therapists on a multidisciplinary rehabilitation programme for persons with brachial plexus injuries. Disabil Rehabil. 2019;41(12):1427-1434. 